\title{
EVALUATION OF A NOVEL UAV-BORNE TOPO-BATHYMETRIC LASER PROFILER
}

\author{
G. Mandlburger ${ }^{\text {a, }}$, M. Pfennigbauer ${ }^{\text {b }}$, M. Wieser ${ }^{\text {a }}$, U. Riegl ${ }^{\text {b }}$, N. Pfeifer ${ }^{\text {a }}$ \\ a TU Wien, Department of Geodesy and Geoinformation, Gusshausstr. 27-29, 1040 Vienna, Austria \\ (gottfried.mandlburger, martin.wieser, norbert.pfeifer)@geo.tuwien.ac.at \\ ${ }^{\mathrm{b}}$ RIEGL Research Forschungsgesellschaft mbH, Riedenburgstr. 48, 3580 Horn, Austria - (mpfennigbauer, uriegl)@ riegl.com
}

Commission I, ICWG I/Vb

KEY WORDS: Laser Bathymetry, UAV, Profiler, Shallow water mapping, Hydraulic roughness

\begin{abstract}
:
We present a novel topo-bathymetric laser profiler. The sensor system (RIEGL BathyCopter) comprises a laser range finder, an Inertial Measurement Unit (IMU), a Global Navigation Satellite System (GNSS) receiver, a control unit, and digital cameras mounted on an octocopter UAV (RiCOPTER). The range finder operates on the time-of-flight measurement principle and utilizes very short laser pulses $(<1 \mathrm{~ns})$ in the green domain of the spectrum $(\lambda=532 \mathrm{~nm})$ for measuring distances to both the water surface and the river bottom. For assessing the precision and accuracy of the system an experiment was carried out in October 2015 at a pre-alpine river (Pielach in Lower Austria). A $200 \mathrm{~m}$ longitudinal section and 12 river cross sections were measured with the BathyCopter sensor system at a flight altitude of 15-20 m above ground level and a measurement rate of $4 \mathrm{kHz}$. The 3D laser profiler points were compared with independent, quasi-simultaneous data acquisitions using (i) the RIEGL VUX1-UAV lightweight topographic laser scanning system (bare earth, water surface) and (ii) terrestrial survey (river bed). Over bare earth the laser profiler heights have a std. dev. of $3 \mathrm{~cm}$, the water surface height appears to be underestimated by $5 \mathrm{~cm}$, and river bottom heights differ from the reference measurements by $10 \mathrm{~cm}$ with a std. dev. of $13 \mathrm{~cm}$. When restricting the comparison to laser profiler bottom points and reference measurements with a lateral offset below $1 \mathrm{~m}$, the values improve to $4 \mathrm{~cm}$ bias with a std. dev. of $6 \mathrm{~cm}$. We report additionally on challenges in comparing UAV-borne to terrestrial profiles. Based on the accuracy and the small footprint $(3.5 \mathrm{~cm}$ at the water surface) we concluded that the acquired 3D points can potentially serve as input data (river bed geometry, grain roughness) and validation data (water surface, water depth) for hydrodynamic-numerical models.
\end{abstract}

\section{INTRODUCTION}

Airborne Laser Bathymetry (ALB), an active remote sensing technique for capturing shallow water areas using green laser light, has rapidly evolved in the recent years. Whereas coastal mapping is the main application of ALB, state-of-the art sensors also enable surveying of smaller inland water bodies and especially clear gravel-bed rivers (Hilldale and Raff, 2008; Kinzel et al., 2013; Fernandez et al., 2014; Mandlburger et al., 2015a). This was mainly made possible by two facts: (i) the increased measurement rate lead to a much higher sampling density (dozens of points per $\mathrm{m}^{2}$ ), and (ii) by narrowing the beam divergence the laser footprint diameter on the surface was brought down from $2-5 \mathrm{~m}$ to approximately $0.5 \mathrm{~m}$ for a standard flying height of 500-600 m above ground/water level (Fernandez et al., 2014; Doneus et al., 2015). With those mission parameters capturing of small to medium rivers (width: 5-25 m, depth: 0$4 \mathrm{~m}$ ) became feasible. One of the main advantages of ALB is the areal coverage with homogeneous point density which is independent of the water depth as this is the case, e.g., for sonar systems (Guenther et al., 2000). Another advantage compared to both sonar-based and terrestrial mapping lies in the fact that no contact with the water body and the alluvial environment is necessary which is especially beneficial for capturing ecologically sensitive areas (Irish and Lillycrop, 1999) and dangerous river sections (cliffs, currents, etc.). The same argument applies for shallow water capturing techniques based on passive imagery (Marcus and Fonstad, 2008) either by exploiting spectrally based information (Legleiter, 2012) or via two-media-photogrammetry (Westaway et al., 2003), but image based methods often fail in case of (overhanging) riparian vegetation whereas ALB can potentially penetrate the vegetation through small openings in the leaf structure (Mandlburger et al, 2015a).

However, the planar mapping resolution of ALB is limited by the size of the laser footprint which, in turn, is constrained by eye safety considerations (Guenther et al., 2000; Fernandez et al., 2014; Doneus et al., 2015). Precise capturing of blocks or boulders with a diameter of less than $50 \mathrm{~cm}$ is therefore impossible with typical ALB setups. Moreover, the additional costs for carrying out a flight mission only pay off for project areas on the reach or catchment level $(5-100 \mathrm{~km})$ whereas, e.g., terrestrial surveying is cost-effective for confined areas of interest (100-1000 m, i.e. stream meander level) provided the area is accessible, the water depth allows wading the river, and the flow velocities are moderate enabling safe surveying. The recent advent of Unmanned Aerial Vehicles (UAV) as carrier platforms for compact laser sensors (Amon et al., 2014; Esposito et al., 2014) has now opened a new perspective also for laser bathymetry to overcome the above mentioned drawbacks w.r.t. limited planar resolution and cost-effectiveness.

Restricting the measurement to ranging, opposed to scanning and ranging, and establishing a sufficient coverage by the flight pattern of an unmanned platform, simple, light weight systems for bathymetric survey can be built, i.e. bathymetric lidar profilers. Using a moderate pulse repetition rate and short pulses, strongly collimated pulses can be generated without compromising eye safety. However, this comes with a reduction

* Corresponding author 
in measurement precision because of the reduced energy. While along a profile the resolution is theoretically increased (no scanning), the question arises, if the precision allows exploiting this in practice.

An experiment was designed and executed to answer the following questions for a specific topo-bathymetric lidar profiler, the RIEGL BathyCopter:

- What accuracy can be reached in practice over different land cover (bare ground, river bed)?

- Can the water surface be detected reliably in order to perform proper range and refraction correction of the bathymetric laser echoes?

- What resolution at the river bed can be reached in practice?

- Can the collected data be used for estimating both the water depth and grain sizes?

The remainder of this manuscript is structured as follows: The new sensor is described in Section 2, the study area and the data acquisition in Section 3 and in Section 4 the acquired data is evaluated. The results are discussed in Section 5.

\section{SENSOR SYSTEM}

The sensor system consists of three major parts: a laser rangefinder, a navigation device consisting of an Inertial Measurement Unit (IMU) and a Global Navigation Satellite System (GNSS) receiver, and a UAV airborne platform.

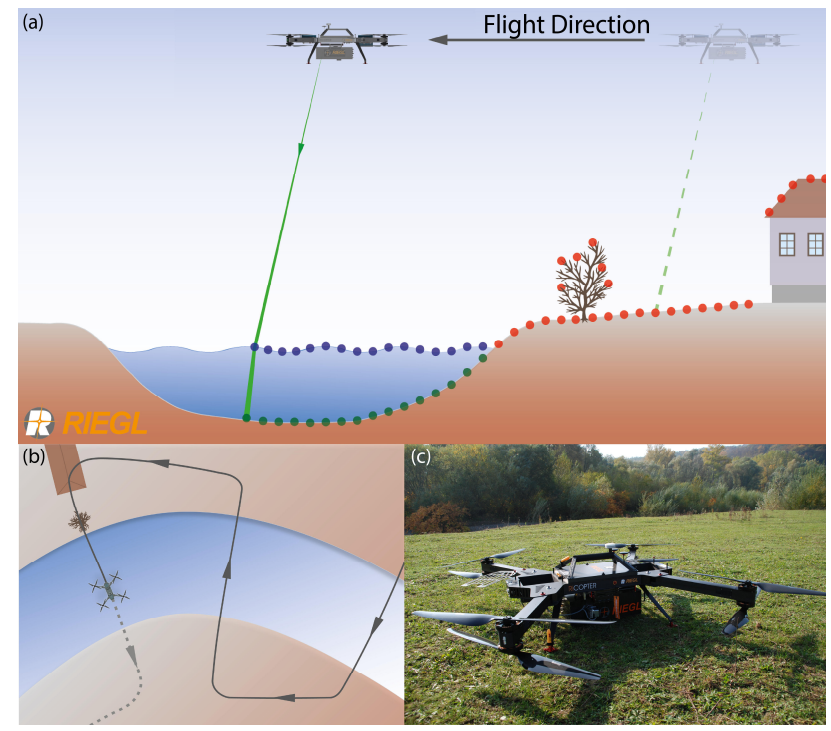

Figure 1: (a) Laser range finder mounted on UAV platform;

(b) Profile oriented data acquisition; (c) BathyCopter ready for take-off at the test site.

\subsection{Laser rangefinder}

The laser rangefinder has a biaxial optical setup with about $5 \mathrm{~cm}$ distance between transmitter and receiver axis. The transmitter is a short-pulsed laser operating at $532 \mathrm{~nm}$ with a pulse repetition rate of $4 \mathrm{kHz}$ and a pulse energy of about $3 \mu \mathrm{J}$. The receiver has an aperture of about $3.5 \mathrm{~cm}$. The echo signal is digitized immediately after opto-electrical conversion and amplification. Full waveform information is stored for every laser shot for the entire range gate. Hence, no triggering, relying on a minimum SNR takes place. This opens up the possibility to perform offline predetection averaging of an adjustable number of pulses.
Determination of the actual measurement range is performed in postprocessing. The laser beam axis is tilted by 8 degrees offnadir which allows receiving enough backscatter to detect echoes from both the water surface and the river bottom for each laser pulse (cf. Figure 1). Knowledge of the exact position of the airwater-interface on a per pulse basis is a prerequisite for proper range and refraction correction of the raw measurements as no areal water surface model can be calculated from measuring points arranged in linear profiles.

\subsection{Navigation system}

The laser rangefinder's optical setup is mechanically tightly coupled to an IMU. Together with an also integrated GNSS of which the antenna is mounted on top of the copter, the navigation system is used to determine the flight trajectory, i.e. position and orientation, with high accuracy and resolution. Hence, origin and direction information can be assigned to every single measurement of the laser rangefinder. The trajectory information is merged with the range results of the measurements to obtain a georeferenced point cloud.

\subsection{Airborne platform}

The laser rangefinder together with the navigation device are mounted on a RiCOPTER platform. The RiCOPTER is an X8 octocopter UAV with a maximum take-off weight of $25 \mathrm{~kg}$ and flight endurance of 30 minutes. It is electrically powered, provides redundant flight control hardware, and can perform autonomous waypoint navigation.

\section{STUDY AREA AND DATA ACQUISITION}

The study area Neubacher Au is located at the tail water of the pre-alpine gravel bed Pielach River (Lower Austria, 48 $12^{\prime}$ '50"' $\mathrm{N}, 15^{\circ} 22^{\prime} 30^{\prime \prime} \mathrm{E}$, WGS 84, cf. Figure 2) and is part of the Natura2000 conservation area Niederösterreichische Alpenvorlandflüsse (Area code: AT1219000). The Pielach River is a medium-sized right side tributary of the Danube and is classified as riffle-pool type with an average gradient in the study reach of $0.39 \%$ (Melcher and Schmutz, 2010). Bed-load sediments are dominated by coarse gravel $(2-6.3 \mathrm{~cm})$ within the active channel and bars. Cohesive sediments in areas of bank erosion lead to steep bank slopes. This, together with the dense understorey vegetation in the riparian forest issues challenges for terrestrial surveys. A more detailed description of the study area can be found in Mandlburger et al. (2015a).

On October 28, 2015, a 200 m longitudinal section and 12 river cross sections of the Pielach River were captured with the BathyCopter sensor system from an altitude of 15-20 m a.g.l. The flight was conducted under good hydrologic conditions (discharge: $5 \mathrm{~m}^{3} \mathrm{~s}^{-1}$, mean annual discharge: $7.16 \mathrm{~m}^{3} \mathrm{~s}^{-1}$, relatively clear water) and moderate weather (bright sky, choppy wind). To ensure cm-precision of the directly georeferenced laser points, thorough static and dynamic initialization of the navigation device was performed on the ground and after take-off following a recommended procedure of the IMU manufacturer (Applanix). For the dynamic initialization the UAV was piloted in arbitrary circular and aft flight manoeuvres for a period of several minutes. The same procedure was also carried out at the end of the flight mission while the laser sensor was still operating (cf. Figure 2). The locations of the longitudinal and cross sections were defined in the flight planning software based on ortho-imagery and depth maps derived from previous ALB missions (Mandlburger et al, 2015a). The waypoints were uploaded to the flight control unit and data capturing was finally conducted by autonomous flight. 


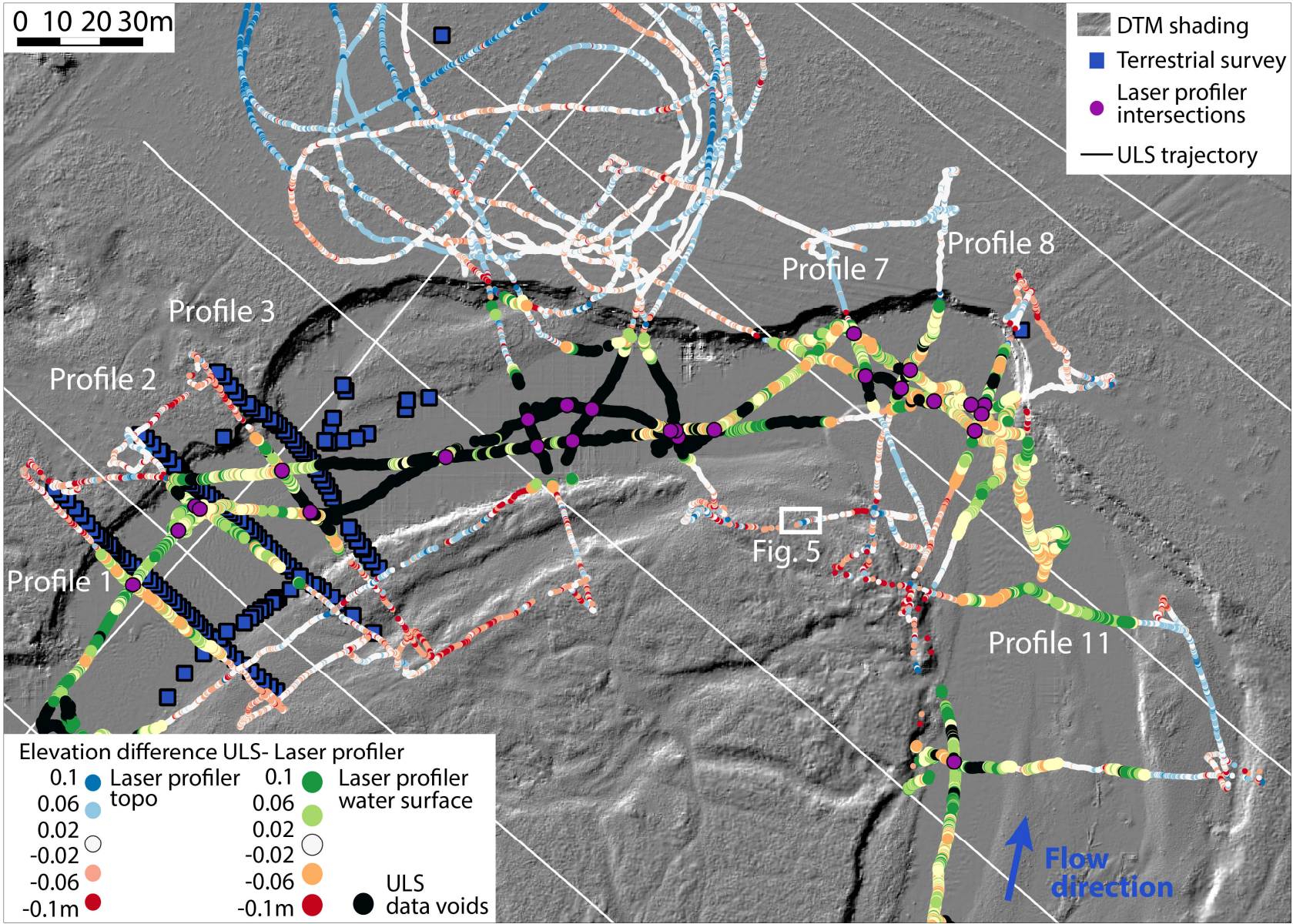

Figure 2. Study area Neubacher Au, background: hill shading, foreground: laser profiler echoes (coloured) and ULS flight path (white), locations of terrestrially surveyed cross sections (blue).

In a second flight following the bathymetric survey the entire study area including the still partially foliated alluvial forest south of the meander bend was captured independently with the RIEGL VUX1-UAV topographic laser scanner (flying altitude: $50 \mathrm{~m}$, flight velocity: $8 \mathrm{~ms}^{-1}$, cf. white lines in Figure 2). On the one hand, this areal survey served for a separate comparison with a leaf-off data set of the same area captured in February 2015 with exactly the same flight planning (Mandlburger et al., $2015 \mathrm{~b}$ ), and on the other hand for assessing the quality of the laser profiler data in the riparian area detailed in Section 4.

To evaluate the quality of the bathymetry data a simultaneous terrestrial survey was conducted. The instrument positions were surveyed in cm-precision with a Leica 1200 GPS (real-time kinematic mode). Based on the pre-defined waypoints three cross sections were marked off at the river bank and in total 170 check points were measured in the submerged area and at the shoreline using a Leica TPS1200 total station. In addition, areal comparison data from an ALB flight in April 2015 (Mandlburger et al, 2015c) captured from $600 \mathrm{~m}$ above ground level with the RIEGL VQ-880-G topo-bathymetric laser scanner mounted on a Diamond DA42 light aircraft were available and served as basis for the quantification of seasonal changes due to fluvial erosion and for estimating the small-scale variability of the riverbed, especially in flow direction.

\section{DATA EVALUATION}

\subsection{Data preprocessing}

In a first step the ULS (VUX1) sensor system was fully recalibrated via rigorous strip adjustment (Glira et al., 2015) making use of the high strip overlap as a consequence of the dense array of flight lines. From the resulting 3D point cloud a high resolution Digital Terrain Model (DTM) with a grid spacing of $15 \mathrm{~cm}$ was derived serving as reference for the comparison with the laser profiler data (bare ground and water surface).

The laser profiler range measurement was performed by applying full waveform analysis in post processing. The raw waveform samples were averaged, filtered and finally the ranges were calculated based on time-of-flight estimation. The range offset originating from the relative position of timing reference and filtered echo signal was determined from the dataset by comparing the bathymetric rangefinder data with the ULS point cloud and, finally, the laser profiler points were corrected accordingly.

\subsection{Accuracy assessment}

The BathyCopter is a topo-bathymetric laser profiler delivering points above, on, and below the water table. The data evaluation started with an accuracy assessment of the profiler bare ground points compared to the ULS DTM. The height deviations are 
plotted in Figure 2 (small circles). Red colours indicate negative height differences (i.e. ULS DTM lower than laser profiler point), white points correspond to areas with small deviations around zero, and blue colour tones are used for positive differences (dark blue: $\geq 10 \mathrm{~cm}$ ). It can be seen that the majority of laser profiler ground points exhibit small deviations and that larger positive and negative height differences are equally distributed. The respective histogram is plotted in Figure $3 \mathrm{a}$ and confirms that the distribution of the deviations is unimodal and symmetric with only a small bias of $-3 \mathrm{~mm}$ and a standard deviation (std. dev.) of $2.9 \mathrm{~cm}$. Apart from the small height bias the deviations do not reveal further systematic effects. A major planar offset across the flow direction can be ruled out as the height differences are mainly in the same order of magnitude at opposite banks. Furthermore, an overall systematic displacement in flow direction is not discernible either, but a time dependent effect is visible (cf. Profile 7 - blue, Profile 8 - white to light red). This basically means that the navigation solution exhibits mediumterm fluctuations in the order of $3 \mathrm{~cm}$, thus, restricting the achievable accuracy to a few $\mathrm{cm}$ as the laser profiler system entirely relies on direct georeferencing.
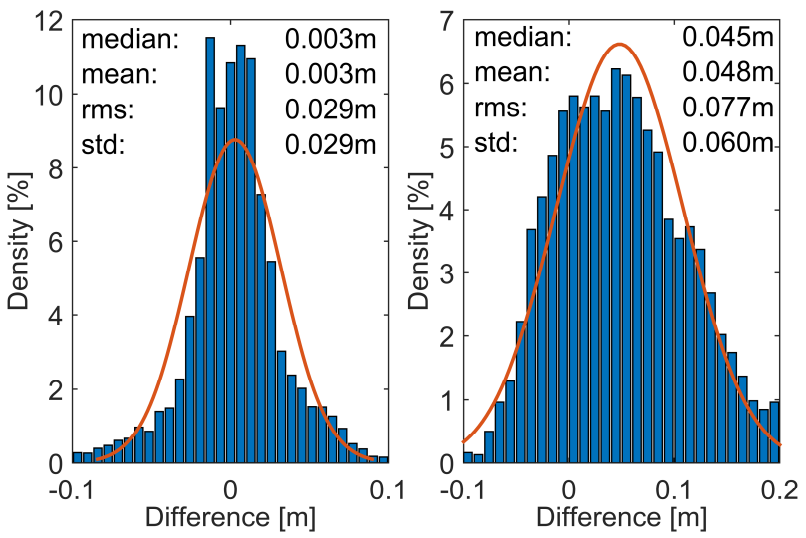

Figure 3. Histogram of elevation differences (ULS DTM minus

laser profiler), red line: PDF of the corresponding normal distribution; (a) bare ground; (b) water surface

Figure $3 \mathrm{~b}$ shows the deviations between the ULS DTM and the laser profiler points at the water surface. In this case the histogram shows a clear positive bias (median: $4.5 \mathrm{~cm}$ ) which can be attributed to the slight penetration of the green laser signal into the top layer of the water column (Guenther et al, 2000). The order of magnitude is in line with the findings in Mandlburger et al. (2013).

As for the higher standard deviation of $6 \mathrm{~cm}$, compared to $3 \mathrm{~cm}$ over land, the short-term variability of the water surface has to be taken into account. Whereas in Mandlburger et al. (2015c) it is stated that the water surface can be treated as static within the study reach under moderate discharge conditions ( $<$ mean flow) when considering a typical ALB footprint size of $60 \mathrm{~cm}$, this is no longer the case for the small footprints of the laser profiler (3.5 cm @15 m altitude) and the ULS system (3 cm @ $60 \mathrm{~m}$ altitude). The effect of the rough water surface can clearly be identified in Profile 11 (cf. Figure 2). The section is divided by a gravel bar into of a rough left part (main channel) and a smooth right part (backwater). Whereas both negative and positive deviations occur in the rough main channel, the deviations in the smooth backwater area constantly positive with a bias in the order of the mean water surface bias. Hence, the calculated $6-\mathrm{cm}$ dispersion is overestimating the actual water level accuracy. A more reliable estimation of the water level accuracy would require a strictly simultaneous acquisition of the water surface, e.g. with independent laser sensors (green, NIR).

To assess the accuracy of the bathymetry, the laser profiler bottom points were compared against check points from terrestrial survey. For three selected cross sections the results are displayed in Figure 4. The individual section plots show the check points (blue) and the laser profiler points (green) along with the ULS points (orange). Furthermore, two additional cross sections derived from the April 2015, VQ-880-G ALB survey enveloping the domain of the respective section are displayed (grey, violet). The latter clearly show (i) the seasonal change of the gravel bed due to fluvial erosion and, even more importantly, (ii) the high variability of the river bed in flow direction. The visual inspection of laser profiler and check points shows good coincidence in some areas and systematic deviations in other areas for all three investigated sections. The deviations are smallest for Profile 1 when considering the entire cross sections, whereas Profile 2 shows very good accordance in the right, southern part of the section and a systematic deviation for the left part (pool). However, both the laser profiler and the check points are consistent when seen individually. Actually, the observed difference can rather be attributed to the small-scale variability of the river bed and to the fact that the laser profiler and the check points are not perfectly aligned in the ground plan (cf. Figure 2). Although, as pointed out earlier, the laser profiler data acquisition was carried out autonomously based on waypoints, and the flight control system ensures minimum deviations from the planned flight path by continuously correcting the sensor positions, still the attitude of the sensor is influenced by turbulences due to varying wind conditions, resulting in displacements of the recorded echoes on the water surface and river bed. On the other hand it was also difficult to exactly position the check points along the planned axis when wading the shallow river bed in the context of the terrestrial survey.

The largest planar deviations between check points and laser profiler points $(4 \mathrm{~m}$ ) apply to Profile 3 (cf. Figure 2 ) where the actual flight path (not displayed in Figure 2) runs perfectly straight between the two datasets. Figure $4 \mathrm{c}$ furthermore reveals that, in the right part of the section, the check points fit much better to the ALB section drawn in violet than to the laser profiler points (green) as this section is well aligned to the check points and the effects of seasonal erosion are small at this bankside. Hence, the numerical comparison of the height differences (laser profiler and ULS vs. terrestrial survey) presented in Table 1 needs to be considered cautiously.

\begin{tabular}{|l|c|c|c|}
\hline \multirow{2}{*}{} & \multicolumn{2}{|c|}{ Laser profiler - TS } & ULS -TS \\
\cline { 2 - 4 } & water & land & \\
\hline Samples & 77 & 53 & 65 \\
Mean & 0.10 & 0.08 & 0.02 \\
Median & 0.07 & 0.06 & 0.01 \\
Std. dev. & 0.13 & 0.17 & 0.05 \\
\hline
\end{tabular}

Table 1. Height differences [m] of laser profiler and ULS compared to check points from terrestrial survey (TS)

The height differences between the ULS dataset and the check points from terrestrial survey (mean: $2 \mathrm{~cm}$, std. dev.: $5 \mathrm{~cm}$ ) are in good accordance with the corresponding comparison of ULS and laser profiler (mean: $0.3 \mathrm{~cm}$, std. dev.: $3 \mathrm{~cm}$ ) and further support the conclusion that the laser profiler data are not affected by a major systematic offset. The $2-\mathrm{cm}$ bias is reasonable as the reflector pole tends to sink in between the gravel grains whereas 
the laser signal is rather reflected from the topmost surface. The gravel roughness also explains the slightly higher dispersion.

The deviations between laser profiler and terrestrial survey data, in contrast, are much higher with a calculated bias of $10 \mathrm{~cm}$ in the river bed and $8 \mathrm{~cm}$ at the bank and standard deviations of $13 \mathrm{~cm}$ and $17 \mathrm{~cm}$, respectively. The main reason for the larger deviations is that the height differences is not measured at the same spot but by comparing the laser profiler height with the height of the nearest check point. The positive bias in both the river bed and river bank comparison are mainly provoked by the left part of Profile 2 and the right part of Profile 3 where the river bottom surface at the measuring position of the laser profiler is clearly higher than the corresponding surface captured by the check points. When restricting the nominal-actual bathymetry comparison to river bed points with a distance to the nearest check point of less than $1 \mathrm{~m}$ the mean deviation drops to $4 \mathrm{~cm}$ with a std. dev. of $6 \mathrm{~cm}$, respectively.

\section{(a) Profile 1}

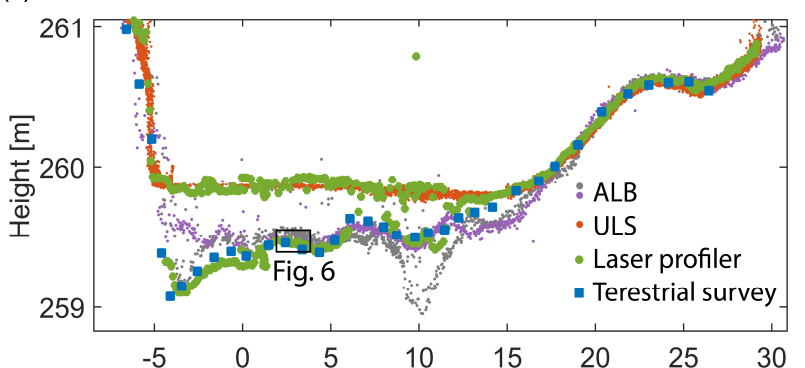

(b) Profile 2

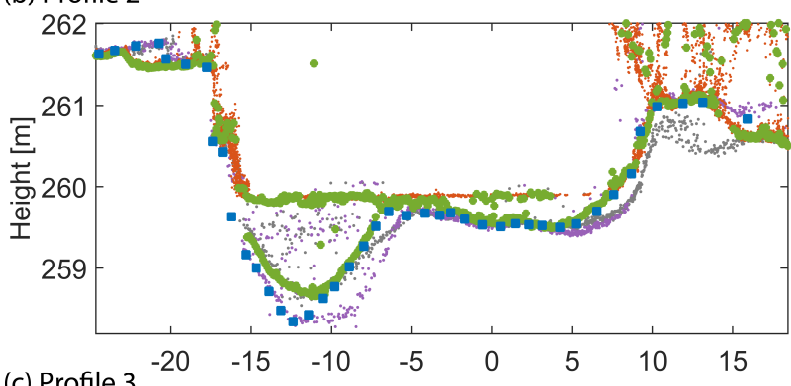

(c) Profile 3

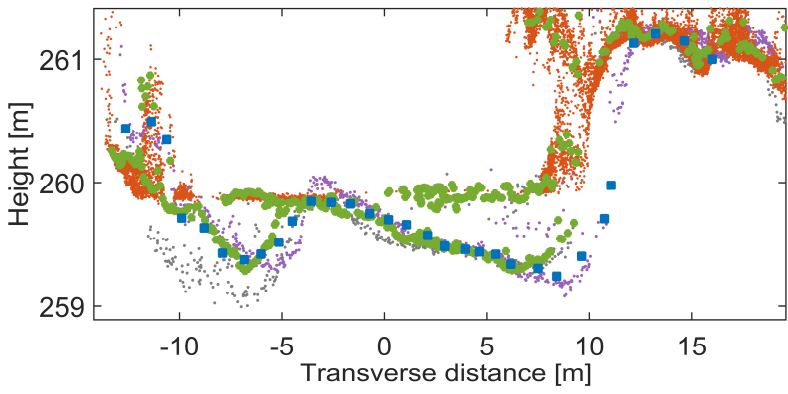

Figure 4: Cross section comparison for Profile 1-3 (a-c); laser profiler (green), ULS (orange), check points (blue), ALB survey (April 2015, grey and violet)

Finally, the laser profiler precision was further assessed by analysing the heights at crossing flight path locations. In total 27 crossings were identified (cf. Figure 2) and the absolute differences feature a mean of $4 \mathrm{~cm}$ and a std. dev. of $3 \mathrm{~cm}$. As this measure comprises the whole set of error sources (sensor position and attitude, ranging, lever arms, boresight angles, etc.) it can be considered a representative measure describing the accuracy potential of the laser profiler.

\section{DISCUSSIONS}

The analysis of the topo-bathymetric laser profiler data carried out so far revealed some challenges and problems but also opened new fields of potential application. Hence, in the following, characteristic features of this novel sensor system are critically discussed.

The combination of bathymetric rangefinder and forward motion of the UAV makes the entire system a topo-bathymetric laser profiler, i.e., the system collects data of both the dry and wetted perimeter along a linear flight path. The main drawback of such a system compared to scanning topo-bathymetric sensors operated from aircrafts is the lack of redundantly acquired data in the overlap area of adjacent flight strips. The latter is used to (i) perform on-the-job calibration of the sensor system via strip adjustment (estimation of boresight angles, range offset and scale, trajectory correction, etc.) and (ii) to assess the fitting precision of the entire flight block based on the zero-difference expectation in smooth strip overlap areas. Today, this is best practice procedure not only for ALB and ALS, but also for ULS (Glira et al., 2015) and was routinely carried out also for the datasets used in this study to ensure optimal sensor calibration and fitting accuracy of the 3D point clouds. The laser profiler, in contrast, entirely relies on direct georeferencing and the positional accuracy of the derived points is tied to the GNSS accuracy. Although no systematic biases of the laser profiler data could be detected compared to (i) the calibrated ULS point cloud (as this was used for the derivation of the profiler's rangefinder offset) and (ii) the terrestrial survey as reference, still a standard deviation of 3-5 $\mathrm{cm}$ is higher by a factor of 2-3 than the respective precision of UAV-borne and airborne scanning systems derived in previous studies for the same area (Glira et al., 2015; Mandlburger et al., 2015a). Whereas scanning systems allow to compensate trajectory errors to a certain extent, this is not possible for a laser profiler. The IMU accuracy is of less concern in this context as the measurement ranges (15-20m) are small.

Within this study the bathymetric accuracy was assessed by comparing the laser profiler points with terrestrially surveyed check points. A major issue hereby was the unsuccessful attempt to acquire the laser profiler points (and the check points) exactly along the predefined path. Whereas the flight control system was successfully guiding the sensor along the planned flight path (cf. the nearly perfect straightness of the ULS trajectory in Figure 2) deviations from the ideal axis of more than $2 \mathrm{~m}$ occurred for the points on the water surface and ground as a result of wind turbulences and corresponding roll motions of the platform. Profile 3, for instance, suffered from a roll angle tilt of 3.5-6 corresponding to a lateral displacement of $1.2-2 \mathrm{~m}$ at a flying altitude of $20 \mathrm{~m}$ a.g.l. A potential compensation for this effect would be the use of either a gyro-stabilized platform or a more sophisticated flight control unit compensating both positional and rotational deviations. The prior would, of course, lead to higher production costs and the latter might lead to varying flight speed and, consequently, to an inhomogeneous point density. But the benefits of a better coincidence with the planned path would surely outperform the drawbacks of an irregular point spacing.

Good alignment of the captured points along the planned axis was discussed in the context of the accuracy assessment so far, but is also highly relevant from an application point of view. When repeatedly surveying bathymetric profiles it is important to capture the profile at the exact location of the previous survey in order to detect changes due to fluvial erosion rather than the small-scale variability of the river bed in flow direction. 
The BathyCopter was designed as a comprehensive sensor for capturing profiles of both the bathymetry and the riparian area with a single instrument and in a single mission. Such data can be used as geometry basis for 1D hydrodynamic-numerical (HN) modelling. Although multi-dimensional HN modelling based on areal bathymetry data from ALB or multi-beam echo sounding can be regarded as state of the art, 1D modelling is still commonplace, especially for large scale flood simulations and the like. Figure 5 shows a vegetated section in the riparian area and demonstrates the ability of the laser profiler to partially penetrate the vegetation layer and provide echoes of the bare ground underneath. The multi-target capabilities of the sensor result from recording and processing the full echo waveform. The vegetation itself is also well contained in the dataset. In addition to the ground surface geometry, this information can be used to characterize the overbank roughness in the hydraulic model (e.g. via calculation of Manning $n$-values derived from vegetation height and density).

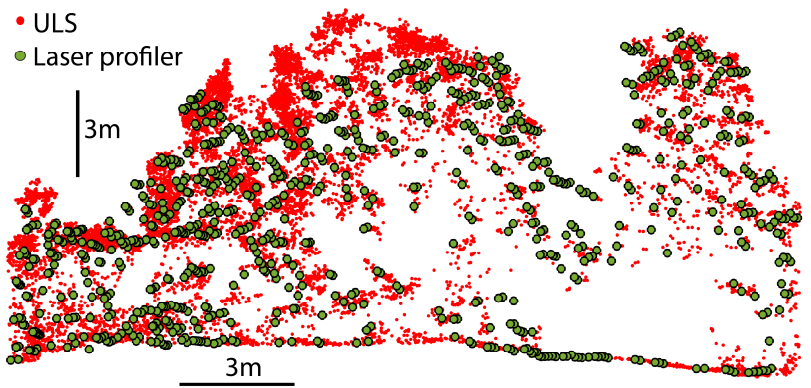

Figure 5: Cross section in vegetated riparian area; laser profiler (green), ULS (red)

Besides the river bed and floodplain geometry HN models require roughness information as second most important input. In this context the composition of the bedload material is of special interest as, e.g., larger boulders provoke a higher roughness than fine sandy material. Within the study area the bedload sediment is dominated by gravel with a grain size of $2-6.3 \mathrm{~cm}$ (Melcher and Schmutz, 2010). While resolutions at such fine scale are not achievably with standard ALB due to the relatively large footprint of typically $>50 \mathrm{~cm}$, the much smaller footprint of the laser profiler $(3.5 \mathrm{~cm})$ together with the low flight velocity (3$4 \mathrm{~ms}^{-1}$ ) and the measurement rate of $4 \mathrm{kHz}$ result in a very small linear point distance on the ground. In general, it is possible to analyse the echo waveform of each individual laser shot resulting in a linear point distance of about $1 \mathrm{~mm}$. However, as it turned out that these data were too noisy, averaging of 100 echo waveforms was employed to increase the signal-to-noise ratio by a factor of 10. This resulted in a net measurement rate of $40 \mathrm{~Hz}$ and a spatial resolution of about $10 \mathrm{~cm}$ (cf. green points in Figure 6). The respective point cloud is smooth and still provides enough geometric details for HN modelling. Averaging 15 echo waveforms leads to a point distance of $1.5 \mathrm{~cm}$. The shape of the corresponding black points of Figure 6 suggests that it is feasible to estimate roughness on the grain size scale together with capturing bathymetry in a single campaign. However, further experiments are needed to verify that the observed variations represent small scale topographic features rather than systematic (periodic) errors.

For this study the range and refraction correction of the raw laser echoes from the water column and river bed was performed based on the ULS DTM. As pointed out in Figure 1 the general strategy for capturing and processing bathymetry with the BathyCopter system is to perform refraction correction for each laser pulse individually. This strategy has the following preconditions: (i) for each laser beam hitting the water body an echo from both the surface and bottom must be recorded and (ii) a horizontal water surface is considered. Data analysis confirmed that $97 \%$ of the river bed points with a depth $>30 \mathrm{~cm}$ feature a corresponding water surface echo. In contrast, only a single echo is recorded in the littoral zone with water depths less than the laser pulse length. In this area proper range and refraction correction can only be performed based on water level heights estimated from the neighbouring profile points. Furthermore, the assumption of a horizontal water surface does not necessarily hold for the small laser footprint of $3.5 \mathrm{~cm}$. Whereas the tilt of the water surface in profile direction can be estimated based on adjacent water surface echoes, the tilt perpendicular to the flight direction cannot be derived from the data. This is a general limitation resulting from the system design (laser profiler) which would be overcome with a UAV-borne topo-bathymetric scanner.

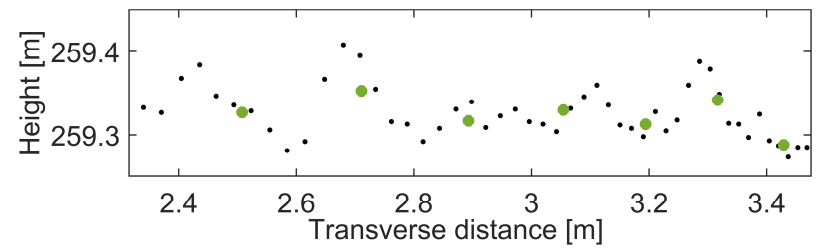

Figure 6: Cross section detail; green/black laser profiler points derived by averaging 100/15 echo waveforms

\section{CONCLUSIONS AND OUTLOOK}

In this article we presented a novel UAV-borne topo-bathymetric laser profiler and reported about an experiment to assess the performance and accuracy of the sensor. The system consists of a laser range finder operating at $\lambda=532 \mathrm{~nm}$, a navigation unit (GNSS, IMU), a flight control system, and optional cameras tightly connected to an octocopter UAV carrier platform. The laser beam is tilted by $8^{\circ}$ off-nadir providing optimal conditions for receiving echoes from both the water surface and the river bed. It could be confirmed that this is the case for $97 \%$ of the laser pulses in areas with a water depth $>30 \mathrm{~cm}$.

Within a test flight 12 cross sections of the near natural pre-alpine Pielach River were captured and compared to the results of quasisimultaneous surveys with (i) a topographic UAV-borne laser scanning system and (ii) a total station. The overall accuracy of the captured topographic and bathymetric laser profiler points strongly depends on the sensor positioning accuracy (GNSS) and was calculated to $3-5 \mathrm{~cm}$ compared the ULS data as reference. Assessment of the bathymetric accuracy was carried out by comparing the laser derived river bed heights with the heights of terrestrially measured river cross sections. The comparison was hampered by the fact that neither the terrestrially surveyed check points nor the laser profiler echoes were exactly aligned to the planned profile axes. The calculated standard deviation of $13 \mathrm{~cm}$ is most likely over estimating the actual error as the visual comparison of the respective cross sections revealed that smallscale river bed variations are responsible for most of the larger deviations between terrestrial and laser bathymetry survey. We conclude that the sensor system would benefit from a stabilization of the laser beam direction.

Whereas the system design as a laser profiler exhibits drawbacks compared to scanning systems concerning (i) system calibration due to a lack of redundant strip overlap area and (ii) refraction correction perpendicular to the flight path, especially the small laser footprint of $3.5 \mathrm{~cm}$ enables new applications as the spatial resolution within a profile is much higher compared to traditional airborne topo-bathymetric scanning. The study showed that the 
water surface could be detected reliably for water depths larger than $30 \mathrm{~cm}$ and that capturing linear profiles of the riparian and submerged area is feasible in a single mission. The latter constitute the main input for 1D HN models. Beyond that, a point spacing of $1.5 \mathrm{~cm}$ on the river bottom (depth: approx. $2.5 \mathrm{~m}$ ) could be obtained and suggests that fine scale roughness estimation of the bottom is feasible with this sensor, but further experiments are needed to confirm this.

\section{ACKNOWLEDGEMENTS}

This study was funded by the Austrian Research Promotion Agency (FFG) COMET-K project "Alpine Airborne Hydromapping - from research to practice".

\section{REFERENCES}

Amon, P., Rieger, P., Riegl, U., Pfennigbauer, M., 2014. Introducing a New Class of Survey-Grade Laser Scanning with Unmanned Aerial Systems (UAS). In: Proceedings of FIG Congress 2014, Kuala Lumpur, Malaysia.

Doneus, M., Miholjek, I., Mandlburger, G., Doneus, N., Verhoeven, G., Briese, C., Pregesbauer, M., 2015. Airborne laser bathymetry for documentation of submerged archaeological sites in shallow water. In: ISPRS Archives of the Photogrammetry, Remote Sensing and Spatial Information Sciences, XL-5/W5, 99 $-107$.

Esposito, S., Mura, M., Fallavollita, P., Balsi, M., Chirici, G., Oradini, A., Marchetti, M., 2014. Performance evaluation of lightweight LiDAR for UAV applications. 2014, IEEE Geoscience and Remote Sensing Symposium, Quebec City, 792 795

Fernandez-Diaz, J., Glennie, C., Carter, W., Shrestha, R., Sartori, M., Singhania, A., Legleiter, C., Overstreet, B., 2014. Early Results of Simultaneous Terrain and Shallow Water Bathymetry Mapping Using a Single-Wavelength Airborne LiDAR Sensor. IEEE Journal of Selected Topics in Applied Earth Observations and Remote Sensing, 7, 623-635.

Glira, G., Pfeifer, N., Mandlburger, G., 2015. Rigorous Strip Adjustment of UAV-Based Laserscanning Data Including TimeDependent Correction of Trajectory Errors. $9^{\text {th }}$ International Symposium on Mobile Mapping Technology, MMT2015, 9-11 December 2015, Sydney, Australia.

Guenther, G.C., Cunningham, A.G., Laroque, P.E., Reid, D.J., 2000. Meeting the accuracy challenge in airborne Lidar bathymetry. In: Proceedings of the 20th EARSeL Symposium: Workshop on Lidar Remote Sensing of Land and Sea, Dresden, Germany.

Hilldale, R., Raff, D., 2008. Assessing the ability of airborne Lidar to map river bathymetry. Earth Surface Processes and Landforms, 33, 773-783.

Irish, J.L., Lillycrop, W.J., 1999. Scanning laser mapping of the coastal zone: the SHOALS system, ISPRS Journal of Photogrammetry and Remote Sensing, 54, 2-3, 123-129

Kinzel, P.J., Legleiter, C.J., Nelson, J.M., 2013. Mapping River Bathymetry With a Small Footprint Green LiDAR: Applications and Challenges. JAWRA Journal of the American Water Resources Association, 49, 183-204.
Legleiter, C.J., 2012. Remote measurement of river morphology via fusion of Lidar topography and spectrally based bathymetry. Earth Surface Processes and Landforms, 37, 499-518.

Mandlburger, G., Hauer, C., Wieser, M., Pfeifer, N., 2015a. Topo-Bathymetric LiDAR for Monitoring River Morphodynamics and Instream Habitats - A Case Study at the Pielach River. Remote Sensing, 7(5), 6160-6195.

Mandlburger, G., Hollaus, M., Glira, P., Wieser, M., Milenkovic, M., Riegl, U., Pfennigbauer, M., 2015b. First examples from the RIEGL VUX-SYS for forestry applications. In: Proceedings of SilviLaser 2015, La Grande Motte, France, 105-107.

Mandlburger, G., Pfennigbauer, M., Riegl, U., Haring, A., Wieser, M., Glira, P., Winiwarter, L., 2015c. Complementing airborne laser bathymetry with UAV-based lidar for capturing alluvial landscapes. In: SPIE Remote Sensing 2015, Toulouse, France, Vol. 9637.

Mandlburger, G., Pfennigbauer, M., Pfeifer, N., 2013. Analyzing near water surface penetration in laser bathymetry - A case study at the River Pielach. In: ISPRS Annals of the Photogrammetry, Remote Sensing and Spatial Information Sciences, II-5(W2), 175-180.

Marcus, W.A., Fonstad, M.A, 2008. Optical remote mapping of rivers at sub-meter resolutions and watershed extents. Earth Surface Processes and Landforms, 33, 4-24.

Melcher, A.H., Schmutz, S., 2010. The importance of structural features for spawning habitat of nase Chondrostoma nasus (L.) and barbel Barbus barbus (L.) in a pre-Alpine river. River Systems, 19, 33-42.

Westaway, R.M., Lane, S.N., Hicks, D.M., 2003. Remote survey of large-scale braided, gravel-bed rivers using digital photogrammetry and image analysis, International Journal of Remote Sensing, 24/4, 795-815. 